\title{
Medical Image of the Month: Malignant Pleural and Pericardial Effusions
}

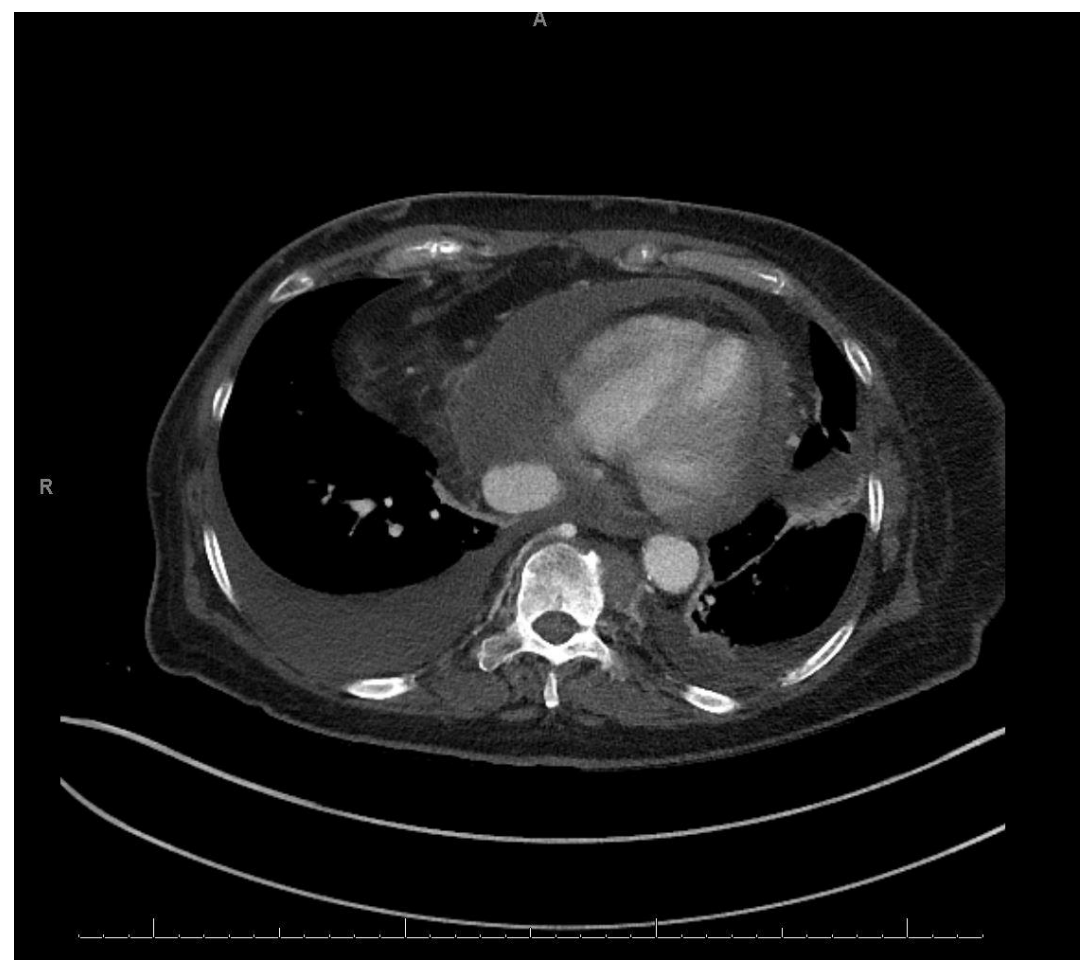

Figure 1. CTA chest axial view showing moderate pericardial effusion, bilateral pleural effusions and anterior mediastinal mass.

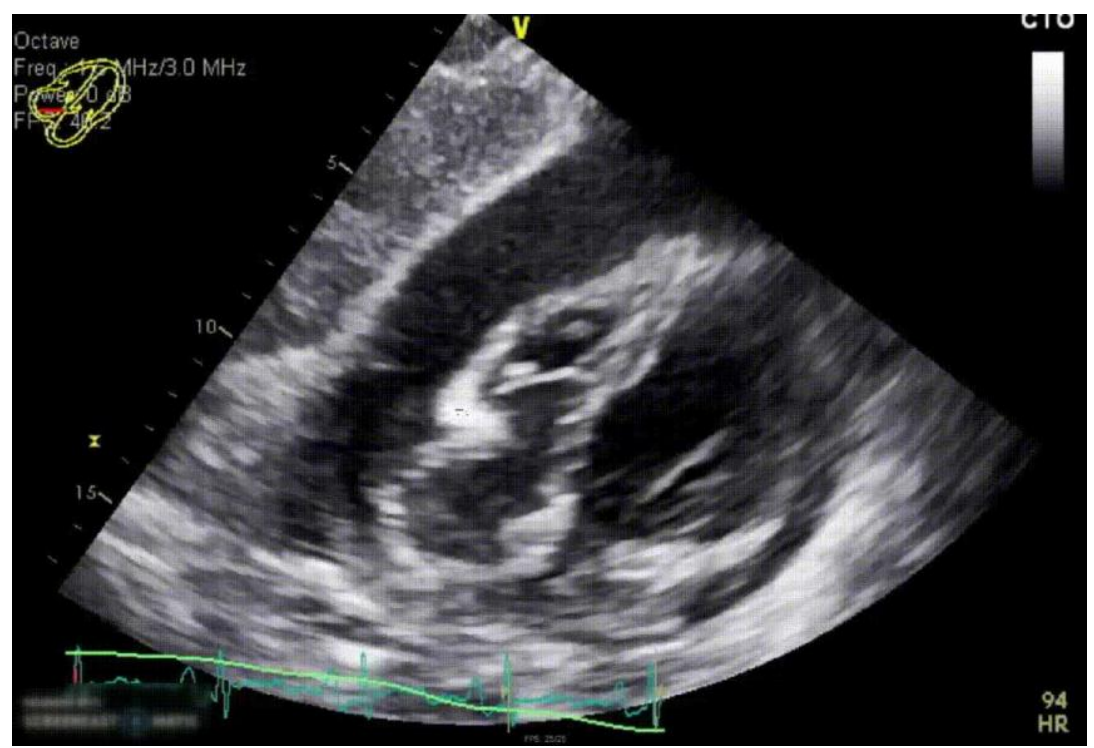

Figure 2. Echocardiography subcostal four-chambered view showing a large pericardial effusion with right ventricular collapse during diastole. 
A 67-year-old woman with a history of presumed thymoma presented to the emergency department with four weeks of progressive shortness of breath and wheezing. CT imaging of the chest on arrival demonstrated a $13.1 \times 8.6 \times 8.2 \mathrm{~cm}$ anterior mediastinal mass with compression of the SVC, pulmonary veins, and right pulmonary artery (Figure 1). A moderate pericardial effusion was also seen. A transthoracic echocardiogram was performed to further evaluate the pericardial effusion, which revealed diastolic collapse of the right ventricle consistent with cardiac tamponade (Figure 2). The patient was taken for urgent pericardiocentesis, which drained $450 \mathrm{cc}$ of sanguineous fluid. Percutaneous biopsy of the mass revealed poorly differentiated carcinoma suspicious for a primary breast malignancy. Cytology of the pericardial fluid did not demonstrate malignancy, however. Cytology of subsequent pleural effusion also was not positive for malignancy, although, both effusions are believed to be related to the malignancy even if no malignant cells were present on analysis.

Malignant pericardial effusions account for $18-23 \%$ of cases, and are one of the most common causes of hemorrhagic effusions. Multiple types of cancers can involve the pericardium; lung cancer is the most common but lymphoma, leukemia, melanoma, and breast cancer are other potentially causative malignancies. Presence of a symptomatic malignant effusion is a poor prognostic indicator with median survival on the order of 24 months after diagnosis, although certain malignancies (e.g. hematologic rather than solid) may have better results (1).

Nathan Coffman MD and Jessica Vondrak MD

Department of Internal Medicine

Banner University Medical Center

University of Arizona

Tucson, AZ USA

\section{Reference}

1. Dequanter $D$, Lothaire $P$, Berghmans $T$, Sculier JP. Severe pericardial effusion in patients with concurrent malignancy: a retrospective analysis of prognostic factors influencing survival. Ann Surg Oncol. 2008 Nov;15(11):3268-71. [CrossRef] [PubMed] 